\title{
Surgery for oligometastatic non-small cell lung cancer: Long-term results from a single center experience
}

\author{
Maria Teresa Congedo, MD, ${ }^{\mathrm{a}}$ Alfredo Cesario, MD, ${ }^{\mathrm{a}, \mathrm{b}}$ Filippo Lococo, MD, ${ }^{\mathrm{a}}$ Chiara De Waure, MD, ${ }^{\mathrm{c}}$ \\ Giovanni Apolone, $\mathrm{PhD},{ }^{\mathrm{d}}$ Elisa Meacci, $\mathrm{MD},{ }^{\mathrm{a}}$ Sergio Cavuto, $\mathrm{MD},{ }^{\mathrm{d}}$ and Pierluigi Granone, $\mathrm{PhD}^{\mathrm{a}}$
}

Objective: The role of surgery in the multimodal therapy for selected stage IV oligometastatic non-small cell lung cancer (NSCLC) is still a forum of open discussion.

\begin{abstract}
Methods: We have retrospectively analyzed the records of 53 patients with oligometastatic NSCLC who had been treated with curative intent in the period January 1997 to May 2010.

Results: The mean age and the male/female ratio were 61 years and 32:21, respectively. A single metastatic lesion was present in $45(84.9 \%)$ subjects, in 2 patients there were 2 different anatomic sites involved, and in 8 patients there were 2 metastases in the same site. The most common involved sites were brain (39), followed by adrenal gland (7), bone (3), vertebrae (3), liver (1), and contralateral supraclavicular lymph node (1). Distant disease was completely resected in 42 patients; 10 patients were treated with exclusive chemotherapy and/or radiotherapy and 1 with local laser therapy. Twenty-nine patients had been administered concurrent chemoradiation in a neoadjuvant setting before the surgical treatment at the lung or both sites (primary/distant). The pulmonary resection was complete (R0) in 42 patients $(79.2 \%$ ). Overall, 1 - and 5-year survivals were $73.1 \%$ and $24 \%$, respectively (median follow-up, 28 months). Median overall survival, local disease-free survival, and distant disease-free survival, estimated using the Kaplan-Meier method, were respectively 19, 72, and 12 months. After stepwise multivariate analysis, the weight loss $(P<.001)$, the completeness of pulmonary resection $(P=.0019)$, and, interestingly, the performance of a positron emission tomography-computed tomography scan in preoperative staging $(P=.05)$ maintained their independent prognostic value as overall survival determinants.
\end{abstract}

Conclusions: Surgical treatment for selected stage IV NSCLC is feasible and safe. Furthermore, good survival can be expected in those patients in whom a complete resection of the primary tumor and radical control of the distant diseases are accomplished. (J Thorac Cardiovasc Surg 2012;144:444-52)

In the majority of patients with lung cancer, the disease is metastatic at the time of diagnosis. According to the most recent data from the American Cancer Society, Surveillance Health and Policy Research, ${ }^{1}$ an estimated 221,130 new cases of lung cancer were expected in 2011, accounting for about $14 \%$ of cancer diagnoses. Historically, metastatic non-small cell lung cancer (NSCLC) has been labeled as stage IV with a single class of M1 disease ${ }^{2}$; in the New Lung Cancer Stage System ${ }^{3}$ a certain degree of variability is described in the M1 status, therefore recognizing a significant heterogeneity within stage IV: M1a indicates positivity for neoplastic cells in the pleural effusion or contralateral

From the Department of General Thoracic Surgery, ${ }^{a}$ Catholic University of Sacred Heart, Rome, Italy; IRCCS San Raffaele Pisana, ${ }^{b}$ Rome; Institute of Hygiene, ${ }^{c}$ Catholic University of Sacred Heart, Rome, Italy; and the IRCCS Arcispedale Santa Maria Nuova, ${ }^{\mathrm{d}}$ Reggio Emilia, Italy.

Disclosures: Authors have nothing to disclose with regard to commercial support.

Received for publication Nov 20, 2011; revisions received April 16, 2012; accepted for publication May 16, 2012; available ahead of print June 19, 2012.

Address for reprints: Maria Teresa Congedo, MD, Department of of General Thoracic

Surgery, Catholic University of Sacred Heart, L.go A. Gemelli 800168 Rome, Italy

(E-mail: t.congedo@libero.it).

0022-5223/\$36.00

Copyright (C) 2012 by The American Association for Thoracic Surgery

doi: $10.1016 /$ j.jtcvs.2012.05.051 lesions (in the lung) and M1b indicates positivity for distant metastases. Within the dismal prognostic setting of stage IV, the M1b class has a very poor prognosis $(1 \%$ at 5 years; overall survival time, 7-11 months). The standard therapeutic approach for stage M1 NSCLC is normally administered with palliative intent on the basis of the invariably very poor outcome, consistently independent from the performance status. Coherently, the common attitude of clinical research efforts in this subgroup of patients with NSCLC has been toward the reduction/control of side/detrimental effects of therapy with specific focus on the quality of life. ${ }^{4}$ However, the subgroup of patients with 1 or 2 distant metastases at the moment of diagnosis and clinical staging (so-called "oligometastatic") probably is encompassed in a prognostic pattern that is different from that of the diffuse distant disease. ${ }^{5}$ Reports from clinical series in which a single brain or adrenal metastasis has been surgically removed would indicate that, indeed, there is justification for a more aggressive, tentatively curative, therapeutic approach in this setting. ${ }^{6-8}$ To the best of our knowledge, very few reports exist about the surgical treatment of oligometastatic NSCLCs, regardless of the metastatic site. ${ }^{6,9}$

The aim of this study is to add knowledge on these subjects by evaluating the feasibility and efficacy of a treatment 


\section{Abbreviations and Acronyms \\ $\mathrm{CT}=$ computed tomography \\ D-DFS $=$ distant disease-free survival \\ ECOG = Eastern Cooperative Oncology Group \\ FDG = fluorodeoxyglucose \\ L-DFS = local disease-free survival \\ LTS $=$ long-term survival \\ NSCLC $=$ non-small cell lung cancer \\ PET = positron emission tomography}

strategy planned with curative intent performed on all sites of metastatic disease in oligometastatic NSCLCs, in addition to the radical surgical treatment of the primary tumor. In this group of patients we aimed at evaluating survival and identifying prognostic factors influencing overall survival and local and distant recurrence.

\section{PATIENTS AND METHODS}

This is an observational study that does not include any experimental treatment. The analysis has been realized on prospectively collected data within the setting of standard clinical practice. Relevant notice has been provided to local ethical committees. Based on the information available from the clinical records, demographic and clinical features were collected and taken into consideration in the statistical analysis. Follow-up information was obtained from our clinical database and from the hospital administrative records. All patients had at least 6 months of follow-up. Patients were censored for either relapse or death. Pretreatment evaluation included patient history, physical examination, lung function tests, baseline hematochemical tests, and computed tomographic (CT) scan of the chest, abdomen, and brain. Histologic confirmation was obtained through standard cytohistology on material from biopsy specimens obtained during fiberoptic bronchoscopy and/or CT-guided fine needle aspiration. Nuclear magnetic resonance imaging of the brain was performed only in nonunivocal cases or to confirm a feature suggestive of metastatic pattern on a CT scan; radiologically suspected N2 disease was always pathologically proven via mediastinoscopy or endoscopic/endobronchial ultrasound endoscopy. A bone radionuclide study was performed in all patients with NSCLC up to the moment when an 18-fluorodeoxyglucose (FDG) positron emission tomographic (PET) scan was routinely performed in addition to other standard staging procedures (as of June 2005). In the preoperative staging, we defined "oligometastatic" disease as a surgically resectable NSCLC primary tumor with 1 or 2 metastatic lesions that were considered, as well, to be radically resectable. Inspired by other similar experiences, ${ }^{9}$ a dedicated multidisciplinary team composed of medical and radiation therapy oncologists, pneumonologists, radiologists, anesthesiologists, and thoracic surgeons established the eligibility criteria; these criteria were (1) local disease and metastatic lesion confined in 1 or 2 sites, both considered radically curable; (2) optimal general clinical condition (evaluated as Eastern Cooperative Oncology Group [ECOG] 0 or 1); (3) no history of other malignant disease; and (4) age less than 76 years. At the moment of surgery, all patients had given their informed consent for the use of data for research purposes, among the other procedures. Surgical criteria were designated as follows: (1) extent of the resection, no less than lobectomy (otherwise considered oncologically inadequate); (2) pneumonectomy, indicated only if less extensive resections were deemed inadequate for completeness; (3) systematic mediastinal lymph node dissection ${ }^{10,11}$ planned and performed in all cases; (4) incompleteness of resection/control at the metastatic site(s): exclusion from the study. Pathologic stage was assigned according to the International Staging System for Lung Cancer $1997^{2}$ and, accordingly, the resection was considered complete if the proximal resection margins and the highest mediastinal node were microscopically free of tumor. In the period between January 1997 and December 2010, a total of 1847 patients underwent major pulmonary resection for NSCLC at the Department of Thoracic Surgery of Catholic University of Rome, Italy. Among these, based on the criteria illustrated above, the relevant data of 53 patients with oligometastatic disease $(2.8 \%)$ were used for the purposes of this study. As well, with respect to the surgical and pathologic features relevant to the analysis reported herein, we collected data on (1) the extent of resection, (2) the pathologic staging, (3) the completeness of resection, (4) the type of mediastinal involvement, and (5) the 30-day morbidity and mortality rates. Follow-up was carried out along standard procedures. Duration of follow-up, survival, and time to first recurrence were available for all patients. For the purpose of the overall survival assessment, time $0\left(\mathrm{~T}_{0}\right)$ was arbitrarily selected as the date of last intervention on the primary lung cancer or on the metastatic lesion and the terminal event $\left(T_{\text {end }}\right)$ was death or status for survivors.

\section{Statistical Analysis}

Survival curves were obtained using the Kaplan-Meier method. Survivals were calculated by the Kaplan-Meier method and compared by the log-rank and the Breslow tests. The independent variables that were tested in the univariate analysis were as follows: weight loss $(>10 \%$ in the last 6 months before treatment), ECOG score, PET-CT, clinical $\mathrm{T}$ stage (0-1 vs $2-4)$ and clinical $\mathrm{N}$ stage (0 vs $1-2)$, metastatic site, number of metastatic sites and number of metastatic lesions, type of treatment for metastatic disease, whole brain radiation, neoadjuvant therapy, histology, pathologic T stage (0-1 vs $2-4)$ and pathologic $\mathrm{N}$ stage ( 0 vs 1-2), surgical completeness, and adjuvant therapy. The univariate analysis was carried out on Kaplan-Meier curves via the application of the Breslow and log-rank tests. Three different end points were analyzed: 5-year overall survival (LTS), defined as the percentage of patients alive at 5 years after the last treatment (on primary or distant site); 5 -year local disease-free survival (L-DFS), defined as the percentage of patients alive without local recurrence of disease at 5 years after the last treatment (on primary or distant site); and 5-year distant disease-free survival (D-DFS), defined as the percentage of patients alive without distant recurrence of disease at 5 years after the last treatment (on primary or distant site). All the variables showing a possible association with survival $(P<.1)$ at the univariate analysis were entered in a multivariate analysis (Cox proportional hazards model) to identify independent prognostic factors. A backward elimination approach was used to obtain hazard ratios with $95 \%$ confidence intervals. SPSS 12.0 software for Windows (SPSS, Inc, Chicago, Ill) was used to perform the analysis.

\section{RESULTS}

\section{Preoperative Staging and Metastatic Pattern}

Results characteristics of the entire group are reported in Table 1 and summarized herein: 40 cases of adenocarcinoma, 8 of squamous cell carcinoma, and 5 of undifferentiated carcinoma. Thirteen patients had "chest only" stage III disease, of whom 3 had T4 disease ( 2 patients with a satellite nodule on the same lobe and 1 patient with pulmonary artery infiltration). Most of the cases were N0 (39/53, $73.6 \%$ ); 10 cases were N2 and 4 N1. Sixteen patients (enrolled in the study from June 2005) underwent PET-CT scan at preoperative diagnostic workup evaluation. In all patients a pathologic uptake of the metastatic lesions was detected. In $45(84.9 \%)$ patients a solitary metastasis was 
TABLE 1. Results characteristics of the entire group

\begin{tabular}{lc}
\hline \multicolumn{1}{c}{ Variables } & $\begin{array}{c}\text { Absolute frequency } \\
\text { (relative frequency \%) }\end{array}$ \\
\hline Age & $61($ range, $26-74)$ \\
M/F & $32 / 21$ \\
ECOG status & \\
0 & $36(67.9)$ \\
1 & $17(32.1)$ \\
Weight loss & $8(15.1)$ \\
Stage cT & \\
1 & $19(35.8)$ \\
2 & $21(39.6)$ \\
3 & $10(18.9)$ \\
4 & $3(5.7)$ \\
Stage cN & \\
0 & $39(73.6)$ \\
1 & $4(7.5)$ \\
2 & $10(18.9)$ \\
Side & \\
Left & $24(45.3)$ \\
Right & $29(54.7)$ \\
Histology & \\
Adenocarcinoma & $40(75.5)$ \\
Squamous carcinoma & $8(15.1)$ \\
Not differentiated & $5(9.4)$ \\
One single metastasis & $45(84.9)$ \\
Two metastases & $8(15.1)$ \\
Metastasis location & \\
Brain & $39(70.9)$ \\
Adrenal gland & $8(14.5)$ \\
Bone & $3(5.5)$ \\
Vertebrae & $3(5.5)$ \\
Liver & $1(1.8)$ \\
Contralateral Supraclavicular node & $1(1.8)$ \\
Two sites & $2(3.8)$ \\
\hline ECOG, Eas &
\end{tabular}

ECOG, Eastern Cooperative Oncology Group.

detected and in $8(15.1 \%)$ there were 2 metastatic lesions. The most common metastatic site was brain $(\mathrm{n}=39)$ followed by adrenal gland $(\mathrm{n}=8)$, bone $(\mathrm{n}=3)$, vertebra $(\mathrm{n}=3)$, contralateral supraclavicular lymph node $(\mathrm{n}=1)$, and liver $(\mathrm{n}=1)$. In particular, among 39 patients with brain metastases, 29 underwent surgical radical excision, whereas in the remaining 10 patients we performed radiosurgery in accordance with the neurosurgeon's opinion. Moreover, all the patients $(\mathrm{n}=8)$ with adrenal gland metastases underwent surgery with or without adjuvant therapy; of 3 patients with bone metastases, 2 underwent a radical bone resection (1 humeral resection and 1 rib resection performed at the same time as the NSCLC surgical procedure), whereas in 1 metastatic lesion located in the left anterior iliac spine we opted for a more conservative treatment (highly conformal irradiation). Similarly, 2 of 3 patients with vertebral lesions were surgically treated (en bloc vertebral resection plus reconstruction) and the remaining patient underwent radiotherapy alone. Finally, both patients with liver $(\mathrm{n}=1)$ and contralateral supraclavicular lymph nodal metastases $(\mathrm{n}=1)$ were surgically treated. Only 2 patients had 2 metastatic sites: a 39-year-old man (liver plus bone) and a 46-year-old woman (bone plus adrenal).

\section{Treatment Strategy}

The patients underwent different therapeutic sequences: (1) metastasis treatment, neoadjuvant therapy, and lung surgery in 22 patients $(22 / 53 ; 41.5 \%)$; (2) metastasis treatment and lung surgery in 20 patients $(20 / 53 ; 37.7 \%)$; (3) neoadjuvant therapy, lung surgery, and metastasis treatment in 7 patients $(7 / 53 ; 13.2 \%)$; and (4) lung surgery and metastasis treatment in 4 patients $(4 / 53 ; 7.5 \%)$. The multidisciplinary team, taking into consideration all relevant parameters, planned the therapeutic schedule. In particular, patients with brain metastases were treated at the level of the brain before the lung resection; patients with adrenal gland metastases, instead, underwent lung resection first (this is done according to the principle of preserving the adrenocortical function for the intervention at the level of the lung). A preoperative treatment with the double aim of controlling the disease locally (with "neoadjuvant" intent) and systemically was performed in $29(58.6 \%)$ subjects. Nineteen patients received chemotherapy alone (cisplatin or carboplatin in combination with paclitaxel or etoposide) and 10 patients (N2 disease) were treated with a combination of chemotherapy and concurrent radiotherapy. ${ }^{12,13}$ A significant local control with consequent clinical downstaging was recorded in $17(58.6 \%)$ of these 29 patients.

None of the patients who received neoadjuvant therapy died during the treatment. Therefore, the entire group of patients $(n=29)$ was assessable for treatment-related complications. Toxicity was generally mild and entirely manageable without treatment interruption. The most common encountered significant treatment-related toxicity was myelosuppression. Grade 4 toxicity did not develop in any of them. One patient experienced neurotoxicity, and other 3 treatment-related hospitalizations occurred, 2 for radiation pneumonitis (grade 3) and 1 for neutropenic fever (grade 3).

Surgical pulmonary resections, pathologic findings, and postoperative features are summarized in Table 2. In 3 patients pneumonectomy was needed (1 right-sided). Thirty-day mortality was $1.9 \%$ (1/53) ( 1 case of pulmonary embolism after right pneumonectomy). Severe complications occurred in 5 patients $(5 / 53 ; 9.4 \%)$ : 3 had pneumonia with consequent respiratory failure, 1 had pulmonary embolism (after lobectomy), and 1 had severe bleeding that required reoperation. Minor complications occurred in 11 patients $(11 / 53 ; 20,8 \%)$ : 3 had supraventricular arrhythmias (medically treated), 3 had persistent air leak, 3 had mucus retention (with lobar/sublobar atelectasia treated with daily bronchial aspirations), 1 had spatial-temporal disorientation that resolved without specific therapy, and 1 had a wound infection that required repeated medications. 
TABLE 2. Surgical pulmonary resections, pathologic findings, and postoperative features

\begin{tabular}{lc}
\hline \multicolumn{1}{c}{ Variables } & $\begin{array}{c}\text { Absolute frequency } \\
\text { (relative frequency } \%)\end{array}$ \\
\hline Surgery for metastasis & $42(79.2)$ \\
Pulmonary surgical procedures & \\
Lobectomies & $47(88.7)$ \\
Bilobectomies & $3(5.7)$ \\
Pneumonectomies & $3(5.7)$ \\
Stage pT & \\
0 & $1(1.9)$ \\
1 & $21(39.6)$ \\
2 & $21(39.6)$ \\
3 & $9(17.0)$ \\
4 & $1(1.9)$ \\
Stage pN & \\
0 & $26(49.1)$ \\
1 & $11(20.8)$ \\
2 & $15(28.3)$ \\
3 & $1(1.9)$ \\
R status & \\
R0 & \\
R1 & $42(79.2)$ \\
R2 & $10(18.9)$ \\
Thirty-day mortality & $1(1.9)$ \\
Severe complications & $1(1.9)$ \\
Mild complications & $5(9.4)$ \\
Whuvant therapy & $11(20.8)$ \\
First recurrence pattern (38) brain irradiation & $38(71.1)$ \\
Local & $33(84.6)$ \\
Metastasis & \\
Local and metastasis & $6(15.8)$ \\
& $30(78.9)$ \\
(3) & $2(5.3)$ \\
\hline
\end{tabular}

Pathologic staging was as follows: 23 stage I, 11 stage II, and 19 stage III. In stage III, 15 patients had persistent mediastinal lymph node disease. In $11(11 / 15 ; 73.3 \%)$ the disease was microscopic and not detectable at preoperative radiologic evaluation (only 1 of these patients underwent PET-CT scan in the preoperative evaluation). Finally, 1 patient showed a complete pathologic response to the preoperative treatment and no neoplastic foci were detected at final pathologic examination (pathologic stage 0). In 10 patients $(10 / 53 ; 18,9 \%)$, some residual microscopic remnants of the tumor were found on the bronchial resection margins and/or in the highest mediastinal resected nodes (R1), whereas a grossly incomplete (R2) resection occurred in only 1 patient $(1 / 53 ; 1.9 \%)$. Adjuvant therapy was administered in 38 patients. In particular, all patients with residual disease and with mediastinal lymph node involvement were treated with chemotherapy and/or radiotherapy (if this had not been used before pulmonary resection). Whole brain radiation was applied in 33 of the 39 patients with brain metastases: 5 patients refused the adjuvant treatment and 1 was excluded because of neurologic function.

\section{Recurrence and Long-Term Survivals}

The median duration of follow-up was 28 months with a minimum follow-up of 6 months. Figure 1, A, shows the Kaplan-Meier survival curve for the entire group (median overall survival, 19 months). Median L-DFS and D-DFS were respectively 72 and 12 months (Figure $1, B$ and $C$ ). At the time of the last follow-up, 30 patients (30/53; $56.6 \%)$ had died of disease, $1(1 / 53 ; 1.9 \%)$ died of another cause, $8(8 / 53 ; 15.1 \%)$ were alive with disease, and $14(14 /$ $53 ; 26.4 \%$ ) patients were alive with no evidence of disease.

\section{Prognostic Factors}

Three different Cox regression analyses have been performed with 3 end points: LTS, L-DFS, and D-DFS (Table 3). At univariate analysis, clinical and pathologic staging (cT factor, $\mathrm{cN}$ factor, $\mathrm{pT}$ factor, and $\mathrm{pN}$ factor) seem to be correlated with overall survival, but this evidence is not confirmed at the multivariate analysis. Completeness of resection was confirmed as a strong determinant of survival: the $11 \mathrm{R} 1 / \mathrm{R} 2$ patients $(11 / 53 ; 20.8 \%)$ showed LTS and L-DFS rates significantly worse than those with R0 status (Figure 2, $A$ and $B$ ). At the same time, the 8 patients $(8 / 53 ; 15.1 \%)$ who experienced a weight loss over $10 \%$ had a very poor LTS (median survival time of 6 vs $28 ; P<.001$ ) and D-DFS (median survival time of 3 vs $16 ; P<.001$ ) if compared with the others (Figure 2, $C$ and $D$ ). Local control of disease was heavily influenced by the mediastinal pathologic involvement: the $\mathrm{N}+$ status was a proven prognostic factor for the L-DFS (Figure 3, $A$ ); in this setting we can justify the role of adjuvant therapy after pulmonary resection with a clinically significant beneficial impact on the L-DFS (Figure 3, B). On the other side, the complete control of the metastatic lesion by surgery (more than with radiation or chemotherapy), regardless of the site, is safe and effective and has a beneficial impact on the D-DFS (Figure 3,C). This is particularly true at the brain level if surgery is followed by whole brain radiation (Figure 3,D).

Moreover, we found that a significantly lower median survival time was shown for patients with 2 brain metastases if compared with median survival of patients with single brain metastasis (10 months and 26 months, respectively; Breslow test $P=.056 ; \log$-rank rest $P=.045$ ). Induction therapy is not a significant prognostic factor in this series, very probably owing to the characteristics of the study group (small size, heterogeneous). Interestingly, patients who underwent PET-CT scan during preoperative workup evaluation seem to have a better survival than the others in terms of LTS (log-rank $P=.004)$ and D-DFS (log-rank $P=.015)$ (Figure $4, A$ and $B$ ).

\section{DISCUSSION}

The prognosis of metastatic NSCLC is very poor, with median survival time ranging from 5 to 6 months. ${ }^{14}$ Thanks 


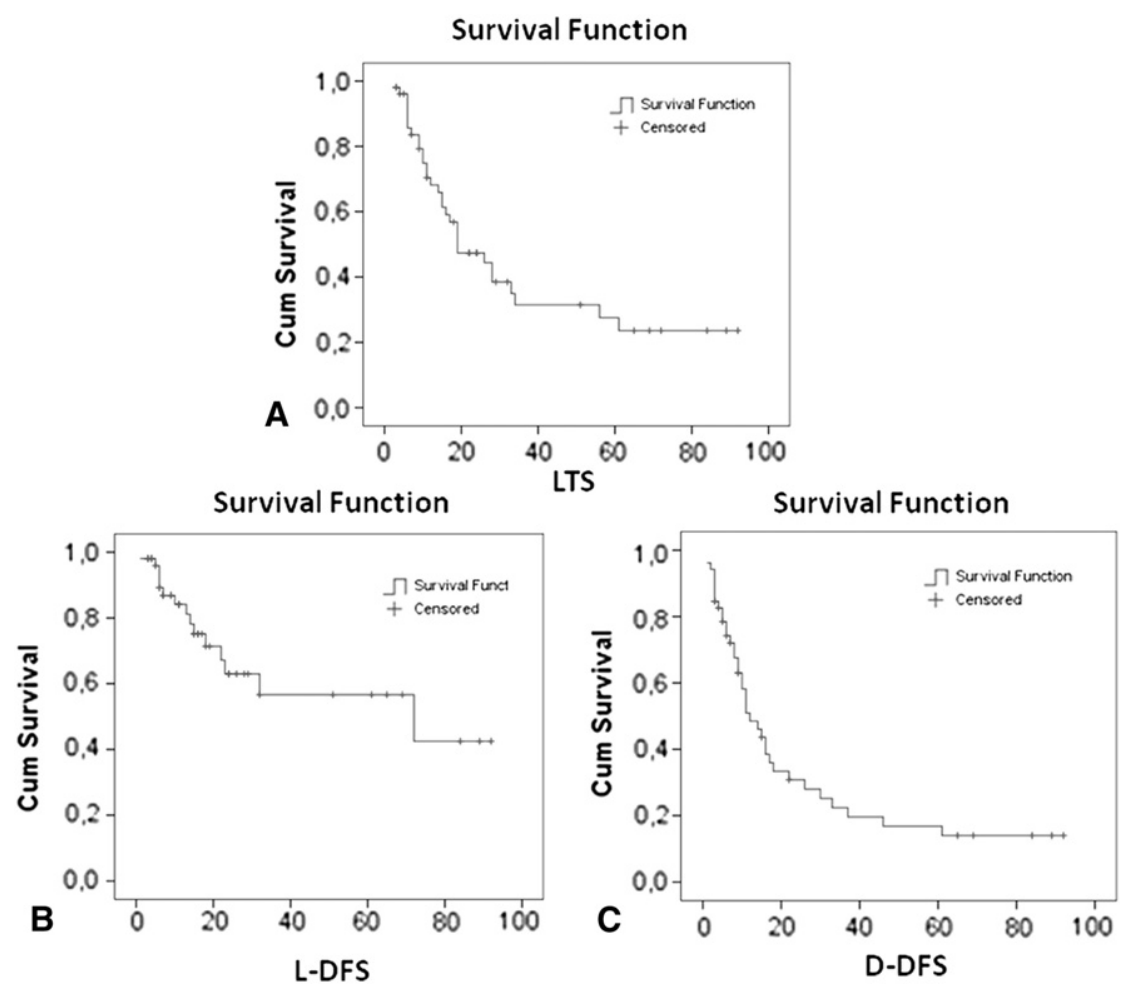

FIGURE 1. Survival: Overall survival of the entire cohort of patients (A), local disease-free survival (L-DFS) (B), and distant disease-free survival (D-DFS) (C). LTS, Long-term survival.

to the availability of more and increasingly effective treatments, the rationale for curing patients with metastatic NSCLC has shifted, over the past 2 decades, from purely palliative to curative intent (disease control and survival) with special attention to the subject's quality of life. ${ }^{4}$ Moreover, sporadic case reports and miniseries have reported

TABLE 3. Cox regression analyses

\begin{tabular}{|c|c|c|c|c|c|c|}
\hline \multirow[b]{2}{*}{ Variables analyzed } & \multicolumn{2}{|r|}{ LTS } & \multicolumn{2}{|r|}{ L-DFS } & \multicolumn{2}{|r|}{ D-DFS } \\
\hline & $\begin{array}{c}\text { Univ. } \\
P\end{array}$ & $\begin{array}{c}\text { Multiv. } \\
{[\text { HR }(95 \% \text { CI })] P}\end{array}$ & $\begin{array}{c}\text { Univ. } \\
P\end{array}$ & $\begin{array}{c}\text { Multiv. } \\
{[\text { HR }(95 \% \text { CI })] P}\end{array}$ & $\begin{array}{c}\text { Univ. } \\
P\end{array}$ & $\begin{array}{c}\text { Multiv. } \\
{[\text { HR }(95 \% \text { CI })] P}\end{array}$ \\
\hline Weight loss* & $P<.001$ & {$[8.01(2.73-23.51)] P<.001$} & NS & NS & $P<.001$ & {$[8.67(2.65-28.40)] P<.001$} \\
\hline cT stage $\dagger$ & $P=.036$ & NS & NS & NS & NS & NS \\
\hline PET-CT scan & $P=.004$ & $\begin{array}{c}{[0.46(0.12-0.98)]} \\
P=.05\end{array}$ & NS & NS & $P=.015$ & {$[0.30(0.09-0.91)] P=.039$} \\
\hline $\mathrm{cN}$ stage $\ddagger$ & NS & NS & $P=.048$ & NS & NS & NS \\
\hline Single metastases & $P=.045$ & NS & $P=.003$ & NS & NS & NS \\
\hline Site of metastases $\S$ & $P=.007$ & NS & NS & NS & $P=.042$ & NS \\
\hline $\begin{array}{l}\text { Surgery for the metastatic } \\
\text { lesion }\end{array}$ & $P=.041$ & NS & NS & NS & $P<.001$ & {$[0.43(0.19-0.98)] P=.045$} \\
\hline Whole brain irradiation & NS & NS & NS & NS & $P=.005$ & {$[0.16(0.03-0.66)] P=.017$} \\
\hline Neoadjuvant Therapy & NS & NS & NS & NS & NS & NS \\
\hline pT stage $\|$ & $P=.006$ & NS & NS & NS & $P=.015$ & NS \\
\hline pN stage & $P=.030$ & NS & $P=.003$ & {$[0.21(0.08-0.89)] P=.008$} & NS & NS \\
\hline $\begin{array}{l}\text { Surgical radicality } \\
\text { (no vs yes) }\end{array}$ & $P<.001$ & {$[4.75(1.87-12.10)] P=.001$} & $P<.001$ & {$[7.43(2.32-17.62)] P<.001$} & $P<.001$ & {$[4.58(1.76-11.92)] P=.002$} \\
\hline Adjuvant therapy & NS & NS & $P=.011$ & {$[4.62(1.71-10.83)] P=.003$} & NS & NS \\
\hline Histology\# & $P<.001$ & NS & $P=.012$ & NS & $P<.001$ & NS \\
\hline
\end{tabular}

LTS, Long-term survival; L-DFS, local disease-free survival; $D$-DFS, distant disease-free survival; Univ., univariate; Multiv., multivariate; HR, hazard ratio; $C I$, confidence interval; $N S$, not significant; PET, positron emission tomography; $C T$, computer tomography. *Over $10 \%$ in the last 6 months; $\nmid \mathrm{cT}$ stage $0-1$ vs cT stage $2-4$; $\ddagger \mathrm{cN} 0$ vs cN + ; $\S$ brain vs other sites; ||pT-stage 0-1 vs pT-stage 2-4; $\uparrow \mathrm{pNO}$ vs pN+; \#not differentiated vs others. 

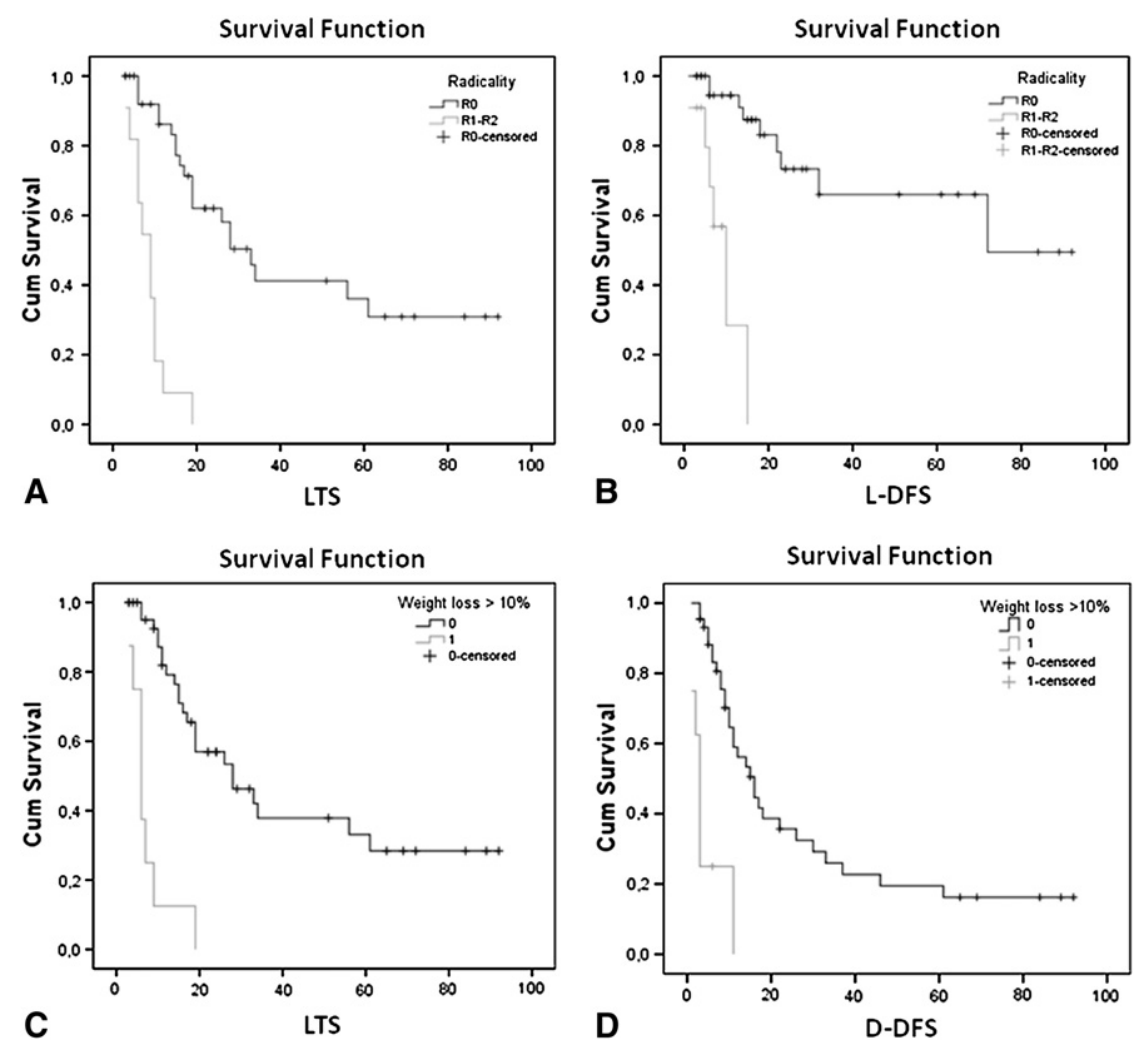

FIGURE 2. Long-term survival (LTS) (A) and local disease-free survival ( $L$-DFS) (B) according with the completeness of resection. Long-term survival (C) and distant disease-free survival (D-DFS) (D) according to the presence of a weight loss ( $>10 \%$ in the last 6 months before treatment).

good survival outcomes in oligometastatic stage IV NSCLCs, and this fact prompted a more aggressive palliation attitude toward and a curative strategy in this select cohort that very probably reflects a less aggressive biologic behavior.

From the viewpoint of safety, these evidences are confirmed by our results on perioperative outcome. Indeed, we could explain our very rewarding 30-day mortality/morbidity rates $(1.9 \%$ and $23.5 \%$, respectively) with the fact that patients were highly selected and the particular strategy of care was supported by an experienced multidisciplinary team.

Safety confirmed, our second aim was to evaluate and confirm evidence from previous reports ${ }^{5-9,15-19}$ on prognostic results of surgical treatment in selected patients with oligometastatic NSCLC. Long-term survival analysis (overall 1- and 5-year survival figures of $73.1 \%$ and $24 \%$, respectively) (Figure 1, A) seem to suggest that this strategy of care is effective.

Following the lines of extreme simplification and according to the background information as available from the literature, we could divide the oligometastatic NSCLCs into 3 groups: (1) cranial, (2) adrenal, and (3) extracranial/extraadrenal. This classification, previously reported by Luketich, ${ }^{15}$ Khan, ${ }^{9}$ and their associates, reflects the typical pattern of distant involvement in oligometastatic NSCLC (brain and adrenal the most common).

\section{Brain}

The brain is the most common site of metastasis from NSCLC, and, in the absence of treatment, the overall median survival from diagnosis is 1 to 2 months. ${ }^{16}$ So far there is no consensus on the standard therapeutic approach regarding primary tumor in patients with synchronous single brain metastasis from NSCLC; in fact, the distant lesion is to be controlled with radical intent (by surgery and or stereotactic radiation therapy). The real usefulness of the combined surgical approach has been analyzed by several groups, mostly in single center (sporadically in the multicenter setting) retrospective studies. In addition to the analysis of the survival and quality of life outcomes, authors have constantly focused their speculation on picking up and validating selection criteria in the preoperative workup to identify the best candidates for this sort of treatment, indeed aggressive and therefore justifiable only in the light of a significant improvement of prognosis. These studies are detailed in Table $4 .^{18,20-25}$

\section{Adrenal}

The detection of a single adrenal metastasis is less frequent than that at the level of the brain, and its incidence is evaluated to be $1.6 \%$ to $4 \%$ of resectable NSCLCs ${ }^{19,26}$; as in the case of the brain, the treatment of adrenal metastasis in patients in whom complete lung resection can be accomplished is still a debated issue. Several 

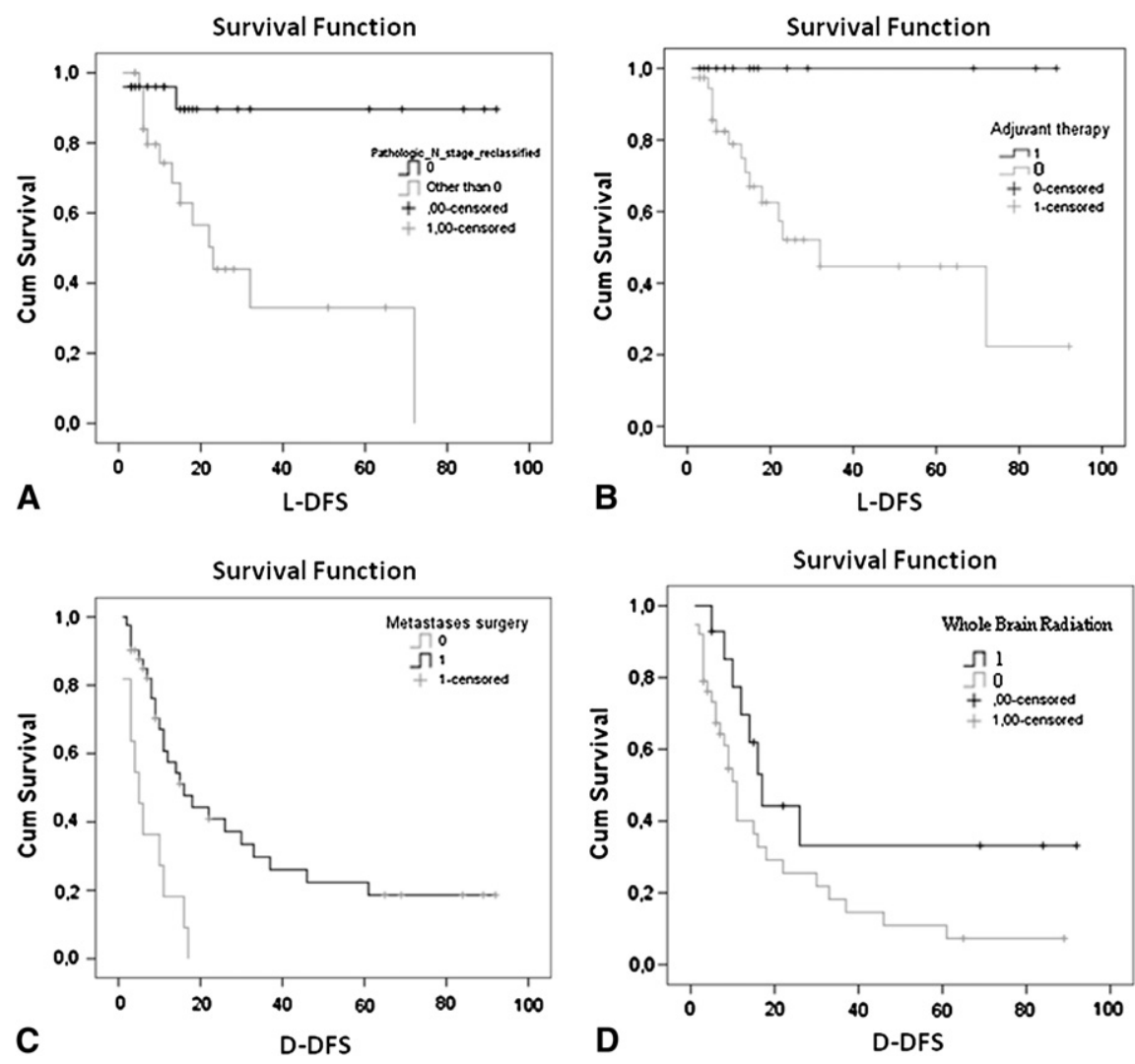

FIGURE 3. Prognostic factors after multimodal treatment for oligometastatic; pathologic $\mathrm{N}$ status (A) and adjuvant therapy (B) are related to local diseasefree survival (L-DFS) whereas the choice of surgical resection of the distant metastases (C) and the execution of whole brain radiation (D) seem to significantly influence the distant disease-free survival $(D-D F S)$.

reports, some dating back 20 years, showed that resection in NSCLC with single adrenal metastasis is feasible and occasionally associated with satisfactory long-term survival. $^{21,25,27,28}$ In the past decade several case series of adrenal resection for NSCLC metastases have been evaluated (details in Table 4) with some rewarding results in terms of long-term survival.

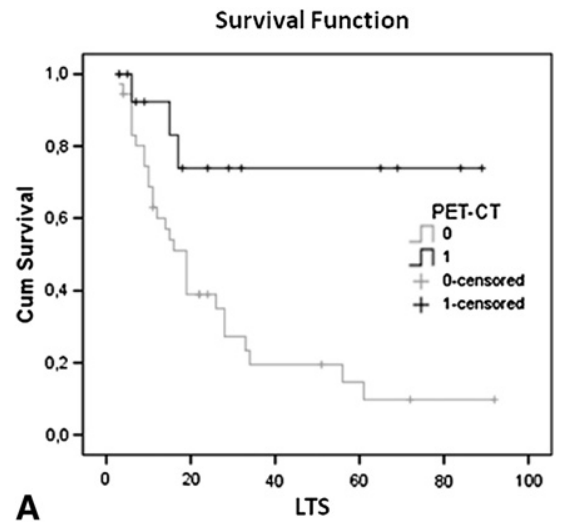

\section{Other Sites}

Other sites are described in rare surgical series (pancreas, ${ }^{29}$ pectoralis muscle, ${ }^{30}$ and leg muscle, ${ }^{31}$ one of the largest being that described by Luketich and associates, ${ }^{15}$ who reported on a group of long-term surviving patients with single extra-adrenal extracranial metachronous metastasis from surgically resected NSCLC. Similarly, Khan and

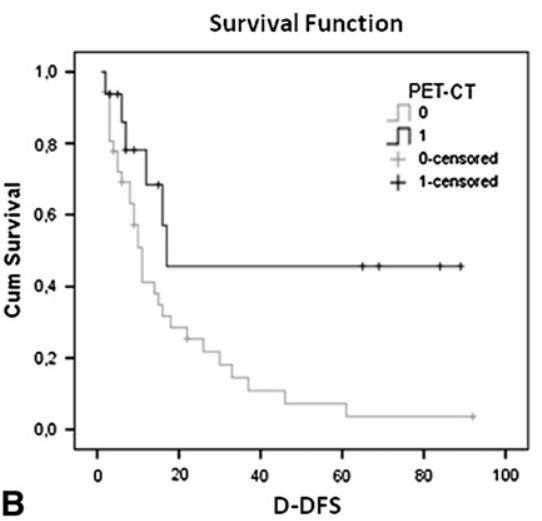

FIGURE 4. The prognostic role of fluorodeoxyglucose positron emission tomographic-computed tomographic (PET-CT) scan: impact on long-term survival (LTS) (A) and distant disease-free survival (D-DFS) (B). 
TABLE 4. Studies of aggressive treatment of metastases

\begin{tabular}{|c|c|c|c|c|c|c|c|}
\hline Studies & No. & $\begin{array}{l}\text { Metastatic } \\
\text { pattern }\end{array}$ & $\begin{array}{c}\text { Radicality } \\
\text { (lung resection) }\end{array}$ & Mortality & Morbidity & LTS & $\begin{array}{l}\text { Prognostic } \\
\text { factors }\end{array}$ \\
\hline$\overline{\text { Billing }(2001)^{20}}$ & 28 & Brain metastases & $100 \%$ & $0 \%$ & $21.4 \%$ & $21.4 \%$ & N0 \\
\hline Porte $(2001)^{21}$ & 43 & Adrenal metastases & $95 \%$ & $0 \%$ & $18.6 \%$ & $14 \%(3 y)$ & None \\
\hline Bonnette $(2001)^{22}$ & 103 & $\begin{array}{l}\text { Synchronous brain } \\
\text { metastases }\end{array}$ & $78 \%$ & $1.9 \%$ & NR & $11 \%$ & $\begin{array}{l}\text { Adenocarcinoma } \\
\text { N0 } \\
\text { T1 }\end{array}$ \\
\hline Granone $(2001)^{18}$ & 30 & Brain metastases & $90 \%$ & $0 \%$ & $0 \%$ (major complications) & $14 \%(3 \mathrm{y})$ & $\begin{array}{l}\text { N0 } \\
\text { Adenocarcinoma }\end{array}$ \\
\hline Iwasaki $(2004)^{23}$ & 70 & Brain metastases & NR & NR & NR & $\begin{array}{l}22 \% \text { ( } 3 \text { y in brain/ } \\
\text { lung surgery) }\end{array}$ & $\begin{array}{l}\text { Adenocarcinoma } \\
\text { NO } \\
\text { CEA }\end{array}$ \\
\hline $\operatorname{Mercier}(2005)^{24}$ & 23 & $\begin{array}{l}\text { Adrenal metastases } \\
\quad \text { (radical lung } \\
\text { resection) }\end{array}$ & - & $0 \%$ & $13 \%$ & $23 \%$ & $\mathrm{DFI}>6 \mathrm{mo}$ \\
\hline $\begin{array}{l}\text { Tanvetyanon } \\
\quad(\text { review, 2008) }\end{array}$ & 114 & Adrenal metastases & $82 \%$ & $0 \%$ & $13 \%-24 \%$ & $25 \%$ & $\begin{array}{c}\text { Metachronous } \\
\text { metastases }\end{array}$ \\
\hline Our series (2012) & 53 & Various sites & $79.2 \%$ & $1.9 \%$ & $23.5 \%$ & $24 \%$ & $\begin{array}{l}\text { R0 } \\
\text { Weight loss } \\
\text { PET-CT }\end{array}$ \\
\hline
\end{tabular}

LTS, Long-term survival; $N R$, not reported; $C E A$, carcinoembryonic antigen; $D F I$, disease-free interval; PET, positron emission tomography; $C T$, computed tomography.

colleagues ${ }^{9}$ in 2006 suggested an "aggressive palliation" to be applied in a class of oligometastatic patients with good performance status and limited weight loss in attempt to control all known sites of disease.

Considering all the pertinent literature as reported above, we can affirm that oligometastatic lung cancer (whatever the site of the metastatic disease) is a still a forum of open discussion. In fact, most of the mentioned reports concluded that combined surgical therapy was the best treatment and probably the gold standard for these selected patients, although the identification of prognostic factors influencing survival remains unclear and the question "who will benefit from aggressive therapy" is unanswered. ${ }^{32}$ We are aware that the present study has the usual limitations of other retrospective monocentric studies (the long duration of patient inclusion and the limited number of patients). Nevertheless, this is one of the largest surgical series of patients with oligometastatic NSCLC with different metastatic sites involved, consecutively treated in a single center. Unlike previous works, all patients enrolled received a complete treatment of both the metastatic lesions (all synchronous at the diagnosis) and the primary pulmonary lesion. Our indications are still not strong enough to support the outlining of guidelines for specific situations because of the great variety of factors (preoperative stage, site of metastasis, modality of metastatic treatment).

\section{The Potential Role of PET-CT/FDG}

One possible solution to this enigma is a screening approach realized through the execution of a PET-CT/FDG of oligometastic patients to rule out those erroneously labeled as such. Very recently, de Pas and colleagues ${ }^{33}$ have hypothesized that this subset of NSCLC patients (PET-CT/ FDG screened) could represent a well-defined population that would indeed benefit from a more aggressive therapeutic strategy than that in the same class of disease but staged without FDG-PET. In our experience we have observed, interestingly, that a comprehensive staging process, with PET-CT/ FDG, could significantly affect the long-term prognosis of this particular class of patients.

\section{CONCLUSIONS}

We can assume that, despite the absence of clear evidence-based guidelines, surgery in highly selected patients with oligometastatic NSCLC represents a feasible, safe, and effective option. In particular, the presence of a single metastasis diagnosed by PET-CT scan, the absence of weight loss of more than $10 \%$, the completeness of resection, and pathologic N0 status were found to be positive predictive factors for long-term outcome. Further investigations are mandatory to clearly confirm and evaluate the real impact of PET-CT scan evaluation on long-term survival in such patients, although specific prospective randomized analysis on a large series of patients is extremely controversial.

\section{References}

1. American Cancer Society. Cancer facts \& figures, 2011. Available at: http:// www.cancer.org/acs/groups/content/@epidemiologysurveilance/documents/ document/acspc-029771.pdf

2. Mountain CF. Revision in the International System for Staging Lung Cancer. Chest. 1997;111:1710-7. 
3. Detterbeck FC, Boffa DJ, Tanoue LT. The new lung cancer staging system. Chest. 2009;136:260-71.

4. Spiro SG, Rudd RM, Souhami RL. Chemotherapy versus supportive care in advanced non-small cell lung cancer: improved survival without detriment to quality of life. Thorax. 2004;59:828-36.

5. Pfannschmidt J, Dienemann H. Surgical treatment of oligo-metastatic non-small cell lung cancer. Lung Cancer. 2010;69:251-8.

6. Daniels M, Wright GM. Complete resection of non-small cell lung cancer and oligo-metastatic brain disease. ANZ J Surg. 2005;75:963-6.

7. Twomey P, Montgomery C, Clark O. Successful treatment of adrenal metastases from large-cell carcinoma of the lung. JAMA. 1982;248:581-3.

8. Raviv G, Klein E, Yellin A, Schneebaum S, Ben-Ari G. Surgical treatment of solitary adrenal metastases from lung carcinoma. J Surg Oncol. 1990;45:123-31.

9. Khan AJ, Metha PS, Zusag TW, Bonomi PD, Faber LP, Shott S, et al. Long term disease-free survival resulting from combined modality management of patients presenting with oligo-metastatic, non-small cell lung carcinoma (NSCLC). Radiother Oncol. 2006;81:163-7.

10. Keller SM, Adak S, Wagner H, Johnson DH. Mediastinal lymph node dissection improves survival in patients with stages II and IIIA non-small cell lung cancer. Ann Thorac Surg. 2000;70:358-65.

11. Wu Y, Huang ZF, Wang SY, Yang XN, Ou W. A randomized trial of systematic nodal dissection in resectable non-small cell lung cancer. Lung Cancer. 2002;36:1-6.

12. Trodella L, D'Angelillo RM, Ramella S. Chemo-radiotherapy in non-small cell lung cancer: the role of gemcitabine. Ann Oncol. 2006;17(Suppl 5):v52-4.

13. D'Angelillo RM, Trodella L, Ciresa M, Cellini F, Fiore M, Greco C, et al. Multimodality treatment of stage III non-small cell lung cancer: analysis of a phase II trial using preoperative cisplatin and gemcitabine with concurrent radiotherapy. $J$ Thorac Oncol. 2009;4:1517-23.

14. Postmus PE, Brambilla E, Chansky K. The IASLC Lung Cancer Staging Project: proposals for revision of the $\mathrm{M}$ descriptors in the forthcoming (seventh) edition of the TNM classification of lung cancer. J Thorac Oncol. 2007;2:686-93.

15. Luketich JD, Martini N, Ginsberg RJ, Rigberg D, Burt ME. Successful treatment of solitary extracranial metastases from non small cell lung cancer. Ann Thorac Surg. 1995;60:1609-11.

16. Zimm S, Warnpler GL, Stablein D. Intracerebral metastases in solid tumor patients. Natural history and results of treatment. Cancer. 1981;48:384-94.

17. Burt M, Wronski M, Arbit E. Resection of brain metastases from non-small-cell lung cancer. Results of therapy. Memorial Sloan-Kettering Cancer Center Thoracic Surgical Staff. J Thorac Cardiovasc Surg. 1992;103:399-410.

18. Granone P, Margaritora S, D'Andrilli A. Non small cell lung cancer with single brain metastasis: the role of surgical treatment. Eur J Cardiothorac Surg. 2001; 20:361-6.
19. Ettinghausen SE, Burt ME. Prospective evaluation of unilateral adrenal masses in patients with operable non-small-cell lung cancer. J Clin Oncol. 1991;9:1462-6.

20. Billing PS, Miller DL, Allen MS, Deschamps C, Trastek VF, Pairolero PC. Surgical treatment of primary lung cancer with synchronous brain metastases. J Thorac Cardiovasc Surg. 2001;122:548-53.

21. Porte H, Siat J, Guibert B. Resection of adrenal metastases from non-small cell lung cancer: a multicentric study. Ann Thorac Surg. 2001;71:981-5.

22. Bonnette P, Puyo P, Gabriel C. Surgical management of non-small cell lung cancer with synchronous brain metastases. Chest. 2001;119:1469-75.

23. Iwasaki A, Shirakusa T, Yoshinaga Y. Evaluation of the treatment of non small cell lung cancer with brain metastases and the role of risk score as a survival predictor. Eur J Cardiothorac Surg. 2004;26:488-93.

24. Mercier O, Fadel E, de Perrot M, Mussot S, Stella F, Chapelier A, Dartevelle P. Surgical treatment of solitary adrenal metastasis from non-small cell lung cancer. J Thorac Cardiovasc Surg. 2005;130:136-40.

25. Tanvetyanon T, Robinson LA, Schell MJ, Strong VE, Kapoor R, Coit DG, et al. Outcomes of adrenalectomy for isolated synchronous versus metachronous adrenal metastases in non-small-cell lung cancer: a systematic review and pooled analysis. J. Clin. Oncol. 2008;26:1142-7.

26. Burt ME, Heelan R, Coit D, McCormack PM, Bains MS, Martini N, et al. Prospective evaluation of unilateral adrenal metastases in patients with operable non-small-cell lung cancer: impact of magnetic resonance imaging. $J$ Thorac Cardiovasc Surg. 1994;107:584-9.

27. Higashiyama M, Doi O, Kodama K, Yokouchi H, Imaoka S, Koyama H. Surgical treatment of adrenal metastasis following pulmonary resection for lung cancer: comparison of adrenalectomy with palliative therapy. Int Surg. 1994;79: 124-9.

28. Wade TP, Longo WE, Virgo KS, Johnson FE. A comparison of adrenalectomy with other resections for metastatic cancers. Am J Surg. 1998;175:183-6.

29. Pericleous S, Mukhlerjee S, Hurchins RR. Lung adenocarcinoma presenting as obstructive jaundice: a case report and review of literature. World J Surg Oncol. 2008;6:120.

30. McKeown PP, Conant P, Auerbach LE. Squamous cell carcinoma of the lung: an unusual metastasis to pectoralis muscle. Ann Thorac Surg. 1996;61:1525-6.

31. Santini M, Vicidomini G, Di Marino MP. Solitary muscle metastasis from lung carcinoma. J Cardiovasc Surg (Torino). 2001;42:701-2.

32. Hu C, Chang EL, Hassenbusch SJ 3rd, Allen PK, Woo SY, Mahajan A, et al. Non small cell lung cancer presenting with synchronous solitary brain metastasis. Cancer. 2006;106:1998-2004.

33. De Pas TM, de Braud F, Catalano G, Putzu C, Veronesi G, Leo F, et al. Oligometastatic non-small cell lung cancer: a multidisciplinary approach in the positron emission tomographic scan era. Ann Thorac Surg. 2007;83:231-4. 